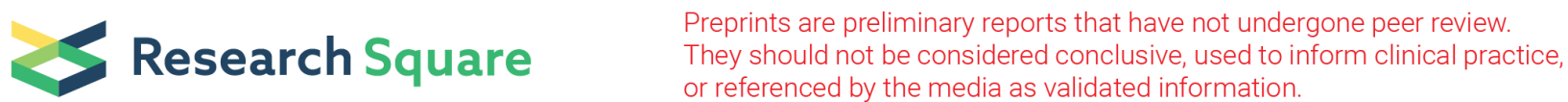 \\ Endometrial Factors Causing Subfertility in Cows
}

\section{Shuichi Matsuyama}

Laboratory of Animal Production Science, Graduate School of Bioagricultural Sciences, Nagoya University, Nagoya 470-0151

\section{Sho Nakamura}

Faculty of Veterinary Medicine, Okayama University of Science, Imabari, 794-8555

\section{Shiori Minabe}

Department of Anatomy and Neurobiology, Graduate School of Medicine, Nippon Medical School, Tokyo

\section{Miki Sakatani}

Division of Animal Feeding and Management Research, Institute of Livestock and Grassland Science, Institute of Livestock and Grassland Science, NARO, Nasushiobara, 329-2793

\section{Naoki Takenouchi}

Livestock and Grassland Research Division, Kyushu Okinawa Agricultural Research Center, National Agriculture and Food Research Organization (NARO), Kumamoto 861-1192

\section{Takuya Sasaki}

Laboratory of Animal Production Science, Graduate School of Bioagricultural Sciences, Nagoya University, Nagoya 470-0151

\section{Hisataka Iwata}

Laboratory of Animal Reproduction, Department of Animal Science, Tokyo University of Agriculture, Kanagawa 243-0034

\section{Koji Kimura ( $\sim$ kimurak@okayama-u.ac.jp )}

Laboratory of Reproductive Physiology, Graduate School of Environmental and Life Science, Okayama University, Okayama 700-8530

\section{Research Article}

Keywords: inadequate uterine environment, embryonic mortality, fertile cows, mitochondrial DNA copy numbers

Posted Date: February 22nd, 2021

DOl: https://doi.org/10.21203/rs.3.rs-200187/v1

License: (c) (i) This work is licensed under a Creative Commons Attribution 4.0 International License. Read Full License 


\section{Abstract}

An inadequate uterine environment is believed to promote embryonic mortality and to result in reproductive failure. We conducted global endometrial gene expression analyses in fertile and subfertile cows to investigate the cause of reproductive failure. The gene expression pattern differed between fertile and subfertile cows; subfertile cows showed a greater number of highly expressed genes than the fertile cows. In particular, the genes encoding ribosomal proteins and mitochondrial oxidative phosphorylation were found among the more highly expressed genes in subfertile cows. The mitochondrial DNA copy number was significantly higher in the endometrium of subfertile cows, whereas the ATP content did not differ between fertile and subfertile cows. Quantitative RT-PCR analysis demonstrated that the expression of $B C L 2 L 13$ was significantly lower in subfertile cows than that in fertile cows. These results suggest that cellular senescence occurs in the endometrium and causes poor-quality mitochondria to accumulate there by reducing mitophagy in subfertile cows. Administration of resveratrol into the uteri of subfertile cows partially increased endometrial BCL2L13 mRNA levels, decreased mitochondrial DNA copy numbers, and allowed for conception following embryo transfer. Collectively, these results suggest that decreased mitophagy in the endometrium of subfertile cows may contribute to infertility.

\section{Introduction}

Decreased reproductive efficiency in cattle, often caused by poor fertility and prolonged calving intervals, reduces the profitability of the cattle industry and decelerates genetic progress. Low fertility is caused by variety of external and internal factors ${ }^{1}$. In addition, as these factors are very complicated, it is difficult to delineate the effects of each individual factor on reproductive function. Previous reports have shown that fertilization rates following artificial insemination (AI) in cattle is over $90 \%{ }^{2}$, suggesting that embryonic death is the main cause of reproductive failures. Several studies have shown that most embryonic deaths occur around the pregnancy recognition period ${ }^{3-6}$. The critical period for embryo death is 7 days after insemination ${ }^{7}$ because during this period, the embryo migrates from the oviduct to the uterus, and the environment surrounding the embryo changes dramatically ${ }^{7}$. Previously, we showed that embryos transferred into the uterus die immediately prior to reaching the pregnancy recognition period ${ }^{8}$, suggesting that the uterine environment during embryo transfer strongly influences embryo viability.

An optimal environment for embryonic development in the uterus is created by the endometrium. The endometrium synthesizes and secretes histotroph into the uterine lumen 9,10 , which is a complex mixture of enzymes, growth factors, cytokines, lymphokines, hormones, transport proteins, nutrients, and exosomes. Such uterine luminal fluid is essential for survival and growth ${ }^{11-15}$, and its composition differs between fertile and subfertile cows ${ }^{7,16}$. During the estrous cycle, endometrial epidermal growth factor concentrations in repeat breeder (RB) cows differ from those in fertile cows ${ }^{16}$ and total protein levels in uterine flushings from RB cows during the 6-8-day period after insemination were significantly lower than those in fertile cows ${ }^{7}$. Additionally, the gene expression patterns in the endometrium of cows have been associated with an optimal uterine environment. Forde et al. revealed that circulating progesterone 
$\left(\mathrm{P}_{4}\right)$ concentrations in the first few days after estrus may influence endometrial gene expression in cyclic heifers ${ }^{17-19}$ and, ultimately, the ability of the uterus to support conceptus development ${ }^{18}$. Therefore, we hypothesized that a functional disorder of the endometrium could lead to early embryonic death and infertility.

Several studies have examined endometrial gene expression in cows with differing fertility $6,20-24$, but any association of endometrial gene expression patterns with functional disorders that cause embryo mortality is still unknown. In the present study, we conducted a global endometrial gene expression analysis on day 7 of the estrous cycle to clarify the differences between fertile and subfertile cows and determine the cause of an inadequate uterine environment for embryo survival and subfertility in cows.

\section{Results}

\section{Endometrial gene expression profiles and plasma $\mathrm{P}_{4}$ concentrations of fertile and subfertile cows}

Plasma $\mathrm{P}_{4}$ concentrations on days 0,5 and 7 did not differ between fertile and subfertile cows (Fig. 1a). Hierarchical cluster analysis of microarray data divided fertile cows into one cluster and subfertile cows into a second cluster (Fig. 1b). Overall, 814 genes were differentially expressed ( $>2$-fold difference, $P<$ 0.05). Of these, 717 were more highly expressed in the endometrium of subfertile cows and the remaining 97 genes were more highly expressed in the endometrium of fertile cows. In particular, the expression levels of the mitochondrial DNA genes $12 S$ and $16 S$ rRNA in subfertile cows were remarkably high (Table 1). Hierarchical cluster analysis of the expression levels of mitochondrial DNA genes, such as for ribosomal RNAs and complexes I-V, similarly divided cows into two clusters based on fertility. Expression levels of nearly all mitochondrial DNA genes (Fig. 1c) and nuclear genes encoding mitochondrial proteins (Fig. 1d) in subfertile cows were higher than those in fertile cows. GO analysis of 717 of the genes expressed more highly in subfertile cows than in fertile cows identified 20 enriched biological processes $(P<0.05 ;$ Table 2$)$. The majority of the 20 terms were related to ribosomal components and biogenesis.

\section{Mitochondrial DNA copy number, ATP content, and SIRT1,PGC-1a,and BCL2L13mRNA expression in the endometrium of fertile and subfertile cows}

To measure any changes to mitochondrial biogenesis and degradation, we determined the mitochondrial DNA copy number, ATP content, and expression profiles of genes associated with mitochondrial biosynthesis and degradation in subfertile and fertile cows. The mitochondrial DNA copy number, calculated as the expression ratio of mitochondria to SOX2 in the endometrium, was significantly higher in subfertile cows than in fertile cows $(P<0.05$, Fig. 2a). However, the ATP content of the endometrium did not differ between fertile and subfertile cows (Fig. 2b). Quantitative RT-PCR (qRT-PCR) analysis showed that expression of sirtuin 1 (SIRT1) and BCL2-like 13 (BCL2L13) mRNAs was significantly lower in subfertile cows than in fertile cows $(P<0.05$, Fig. 3a and $\mathrm{C})$. Peroxisome proliferator-activated receptor gamma coactivator 1-alpha ( $P G C$-1a) mRNA expression was also lower in the endometrial tissues of subfertile cows than in fertile cows, although the difference was not significant (Fig. 3b, $P=0.08$ ). 


\section{Effect of resveratrol administration on SIRT1 and BCL2L13 mRNA expressions, mitochondrial DNA copy number in the endometrium, and pregnancy rate after embryo transfer in subfertile cows}

Administration of resveratrol $(100 \mu \mathrm{M})$ resulted in a non-significant elevation of $B C L 2 L 13$ mRNA levels, although SIRT1 mRNA levels were unaffected (Fig. 4). Mitochondrial DNA copy number in the endometrium of subfertile cows decreased non-significantly following resveratrol administration. BCL2L13 mRNA expression levels and mitochondrial DNA copy number in subfertile cows administered resveratrol were between the values observed for fertile and subfertile cows. Following embryo transfer (ET), two out of five subfertile animals treated with resveratrol conceived, but none of the five subfertile cows that received the vehicle conceived (Table 3). In cows that were not classified by fertility, the pregnancy rates after ET in cows administered resveratrol and vehicle were $75.0 \%$ and $40.0 \%$, respectively. There was a significant difference $(P<0.05)$ in the pregnancy rate between groups $($ Table 3$)$.

\section{Discussion}

In the present study, global gene expression analysis revealed that endometrial genes were differentially expressed in fertile and subfertile cows. Interestingly, a larger number of genes were more highly expressed in subfertile cows than in fertile cows. Moreover, the expression of mitochondrial DNA genes was higher in subfertile cows than in fertile cows. Consistent with these results, the mitochondrial DNA copy number in the endometrium was higher in subfertile cows than in fertile cows. Because the number and quality of mitochondria are maintained by the balance between mitochondrial biogenesis and exclusion $^{25,26}$, a disturbed balance might result in an increased mitochondrial copy number in the endometrium of subfertile cows. Several studies have demonstrated that mitochondrial biogenesis is regulated, at least in part, by PGC- $1 a^{27}$. In addition, members of the mammalian sirtuin family (SIRT1-7) act predominantly as nicotinamide adenine dinucleotide (NAD)-dependent deacetylases and promote mitochondrial biogenesis through PGC-1a activation ${ }^{28}$. The present study has demonstrated that SIRT1 and $P G C$-1a expression levels are lower in the endometrium of subfertile cows than in fertile cows, but that $P G C-1$ a expression is not significantly different. Thus, the high mitochondrial DNA copy number in the endometrium of subfertile cows suggests that a decrease in capacity for mitochondrial exclusion occurred rather than an increase in mitochondrial biogenesis.

Mitochondrial exclusion from cells is controlled by mitophagy-selective autophagy of mitochondria. Mitophagy is believed to be an important mechanism for mitochondrial quality control because it eliminates damaged mitochondria ${ }^{29-32}$, and defects in mitophagy are implicated in the development of neurodegenerative disease ${ }^{33}$, heart failure ${ }^{34}$, cancer $^{35}$, and aging ${ }^{36}$. To date, several autophagy-related genes (Atg) that encode the molecular machinery for mitophagy have been identified. In yeast, Atg32, a mitochondrial outer membrane protein, is indispensable for mitophagy, because it recruits the canonical autophagic machinery to mitochondria through its interaction with Atg8 and Atg11 37,38 . Murakawa et al. demonstrated that BCL2-like protein 13 (BCL2L13) is a functional homolog of Atg32, and is involved in mitochondrial fragmentation as well as mitophagy in mammalian cells ${ }^{39}$. In the present study, BCL2L13 
mRNA expression was lower in subfertile cows than in fertile cows, suggesting that their capacity for mitophagy was reduced. Considering the similar ATP content and the higher mitochondrial DNA copy number in subfertile cows relative to fertile cows, damaged mitochondria accumulate in the endometrium due to reduced mitophagy activity.

Mitochondrial accumulation has been shown to occur in senescent cells ${ }^{40,41}$. Moreover, it has been proposed that the accumulated mitochondria during cell senescence are dysfunctional ${ }^{42}$. In senescent cells, mitophagy activity is reduced in studies in vitro ${ }^{43}$ and in vivo ${ }^{44}$. Taken together, it is possible that cellular senescence occurs in the endometrium of subfertile cows. In addition, in this study, many ribosomal protein genes, which are mainly associated with ribosomal components and biogenesis, were found to be highly expressed in subfertile cows. An increase in ribosomal biogenesis is one feature of senescent cells $s^{45,46}$. Most of the ribosomal protein genes were demonstrated to be upregulated in senescence cells induced by SETD8 depletion ${ }^{45}$. During cell senescence, acceleration of ribosomal RNA transcription and delay in ribosomal RNA processing leads to increased nucleolar RNA content ${ }^{46}$. The subfertile cows in this study expressed a larger number of nuclear genes, and more highly, than fertile cows. Additionally, the genes encoding mitochondrial oxidative phosphorylation (OXPHOS) were remarkably highly expressed in the endometrium of subfertile cows. Increased mitochondrial OXPHOS activity is another feature of cellular senescence ${ }^{47-51}$. From these results, we speculated that cellular senescence occurs in the endometrium and causes attenuation of mitophagy activity in subfertile cows.

Resveratrol, a small polyphenolic compound with antioxidant properties ${ }^{52}$, has been shown to accelerate mitophagy ${ }^{53}$. In our study, BCL2L 13 mRNA expression slightly increased and the mitochondrial DNA copy number slightly decreased in subfertile cows following resveratrol administration, each reaching a point between the values observed for fertile and subfertile cows, although the effects were not significant. These results suggest that resveratrol could increase mitophagy activity in subfertile cows, but not to the level seen in fertile cows. In the present study, the conception rate after ET in cows administered resveratrol was significantly higher than that in cows treated with vehicle. Notably, based on the conception rate of subfertile cows, two out of five subfertile animals treated with resveratrol-but none of the five subfertile cows who received the vehicle-conceived after ET. Considering the effects of resveratrol, it is possible that mitophagy malfunction in the endometrium is a cause of inadequate uterine environments for embryonic survival in cows. However, the small sample size diminishes the capacity of the study to claim any benefit in favor of resveratrol. Larger and well-designed studies are needed to further evaluate the clinical relevance of this drug on improving reproductive outcomes in subfertile cows.

Our results show increased gene expression in subfertile cows, in contrast to the result of previous global transcriptional analyses performed on day 7 of the estrous cycle ${ }^{21,22}$. Additionally, the selection of genes suggested to be associated with fertility ${ }^{21,22}$, did not differ between fertile and subfertile cows in this experiment (Fig S1). These discrepancies between studies might be caused by differences in the biopsy method-uterine biopsy forceps or cytobrush ${ }^{21}$-or animal model ${ }^{22}$ used, although the exact reason has not been clarified. The major cells obtained by each biopsy method would also be different, which might 
explain the varied observed results. Since stromal cells, glandular epithelial cells, and luminal epithelial cells were contained in the biopsy samples of this study (Fig. S2), further studies are required to elucidate which types of endometrial cells exhibit altered mitophagy activity.

Endometrial gene expression has been demonstrated to be altered by circulating $\mathrm{P}_{4}$ levels in the immediate post-ovulation period in heifers ${ }^{17-19}$. However, circulating $\mathrm{P}_{4}$ levels do not explain differential gene expression in the endometrium of fertile and subfertile cows, because plasma $\mathrm{P}_{4}$ concentrations on days 0,5 , and 7 did not differ between groups in this study. In addition, we showed that the gene expression pattern was similar in bovine endometria at $60.1 \pm 4.0$ and $388.9 \pm 8.2$ days after parturition, and only four genes-PGFSII, GPNMB, ATP6VOD2, AMICA1-were differentially expressed. Although the term after calving differed between fertile and subfertile cows in this experiment, our results indicate that it would not affect gene expression in the endometrium of cows. Factors that result in differential expression of fertility-associated endometrial genes should be investigated further.

In summary, this study demonstrated that the gene expression pattern in the endometrium differed between fertile and subfertile cows. Among them, the genes encoding ribosomal proteins and mitochondrial OXPHOS were contained in highly expressed genes in subfertile cows. In subfertile cows, the endometrial mitochondrial DNA copy number was significantly higher, but the ATP content was not different that in fertile cows. Moreover, endometrial BCL2L13 mRNA levels were significantly lower in subfertile cows than in fertile cows. These results suggest that cellular senescence occurs in the endometrium and causes poor-quality mitochondria to accumulate there by reducing mitophagy in subfertile cows. Additionally, administration of resveratrol into the uteri may improve the uterine environment by accelerating mitophagy activity in the endometrium and consequently promoting fertility.

\section{Methods}

\section{Animals}

A total of 139 female multiparous cows were used in this study. The cows were classified as fertile or subfertile according to their reproductive records. Fertile cows were defined as those that experienced pregnancy within three attempts of embryo transfer (ET) or artificial insemination (Al) before biopsy. Subfertile cows were defined as those that failed to conceive following three consecutive ETs or Als, had no detectable abnormalities in their genital tracts, and had apparently normal estrous cycles. During microarray analysis, fertile and subfertile cows were selected based on stricter criteria. Among fertile cows, those that became pregnant following the first ET in the cycles following endometrial biopsy sample collection were defined in the microarray analysis as fertile cows. Among subfertile cows, those that failed to conceive following three consecutive ETs after biopsy without detectable abnormalities in their genital tracts and with apparently normal estrous cycles were defined in the microarray analysis as subfertile cows. Information on age, body weight, body condition score, and parity of experimental animals is shown in Table S1. All cows were fed a grass silage-based diet ad libitum. All experimental procedures involving animals were approved by the Committee for the Care and Use of Experimental 
Animals at NARO (Permission number: 15112008). all methods were conducted according to the relevant guidelines and regulations. All experiments were conducted in according to ARRIVE guidelines.

\section{Experiment 1. Comparison of global gene expression and function in endometria of fertile and subfertile cows}

The day of standing estrous was defined as day 0 . On day 7, several endometrial tissues were obtained from the uterine horn, ipsilateral to the corpus luteum, using uterine biopsy forceps (Integra LifeSciences Corporation; Plainsboro, NJ, USA) in each cow. Three or four biopsy sections, composed of stromal cells, glandular epithelial cells, and luminal epithelial cells (Fig. S2), from each cow were mixed and immediately frozen in liquid nitrogen or suspended in QIAzol reagent (Qiagen; Tokyo, Japan), then stored at $-80^{\circ} \mathrm{C}$ until analysis.

\section{RNA extraction and microarray analysis}

Total RNA was extracted from endometrial samples using the RNeasy Plus Universal Mini Kit (Qiagen) according to the manufacturer's instructions. Total RNA quality and quantity were confirmed using a 2100 Bioanalyzer (Agilent Technologies; Santa Clara, CA, USA) and NanoDrop ND-1000 (Thermo Fisher Scientific; Waltham, MA, USA), respectively. Samples with an RNA integrity number exceeding 6.6. were used for the microarray analysis. Microarray analysis was performed according to the procedure described previously ${ }^{54}$. Samples were labeled using a Quick Amp Labeling Kit, One-Color (Agilent Technologies) in the presence of cyanine-3 (Су3)-CTP, according to the manufacturer's protocol. For microarray hybridization, $1650 \mathrm{ng}$ of Cy3-labeled cRNA was fragmented and hybridized to a bovine $4 \times 44 \mathrm{~K}$ microarray slide (Agilent Technologies; 023647 ) at $65^{\circ} \mathrm{C}$ for $17 \mathrm{~h}$. Slides were scanned using the Agilent G2505C DNA microarray scanner, and background Cy3 signals were corrected using the Agilent Feature Extraction software (version 10.5.1.1). Data were analyzed using GeneSpring GX software (ver.14.9). The raw signal intensity of all probes was subjected to 75th percentile normalization, and the baseline was transformed to the median of all samples. The parameter values for experimental groups were set as "fertile" and "subfertile" cows. Probes were filtered based on their signal intensity values (cutoff $<20 \%$ ), flag values (detected or not detected), and coefficient variation ( $\% \mathrm{CV}<50 \%$ ). For hierarchical clustering analysis, we used the Euclidean distance and average linkage with the relative expression values. Genes that were differentially expressed in fertile and subfertile cows were identified by a threshold of a $>2$-fold change and $P<0.05$ by an unpaired $t$-test. The microarray data were deposited in the Gene Expression Omnibus (GEO) database (accession number GSE107741).

\section{Blood sampling and progesterone assay}

Blood samples were collected on days 0,5 , and 7 for analysis of plasma $\mathrm{P}_{4}$ concentration. Plasma was separated by centrifugation $\left(4^{\circ} \mathrm{C}, 1500 \mathrm{~g}, 30 \mathrm{~min}\right.$ ) and stored at $-30{ }^{\circ} \mathrm{C}$ until further analysis. Plasma $\mathrm{P}_{4}$ concentrations were determined using a double-antibody enzyme immunoassay, as described previously ${ }^{8}$. The assay sensitivity was $0.1 \mathrm{ng} / \mathrm{mL}$ for $100 \mu \mathrm{L}$ plasma samples; therefore, a sample 
yielding a signal below this threshold was assigned a value of $0.1 \mathrm{ng} / \mathrm{mL}$. The intra- and inter-assay coefficients of variation were $5.1 \%$ at $6.3 \mathrm{ng} / \mathrm{mL}$ and $8.5 \%$ at $6.2 \mathrm{ng} / \mathrm{mL}$, respectively.

\section{Assessment of mitochondrial DNA copy number}

The mitochondrial DNA copy number in the endometrial biopsies was determined by quantitative PCR (qPCR) analysis within 2 months of DNA extraction. DNA was extracted from the endometrial biopsies using the DNeasy Blood \& Tissue Kit (Qiagen) according to the manufacturer's instructions. qPCR was performed using the Mx4000 Multiplex Quantitative PCR system (Agilent Technologies) as preciously described ${ }^{55}$. The primer sequences for mitochondria and SOX2 are listed in Table S2. Each reaction mixture consisted of DNA, forward and reverse primers, Brilliant II SYBR® Master Mix (Agilent Technologies), and nuclease-free water in a total reaction volume of $20 \mu \mathrm{L}$. PCR was performed under the following conditions: $95^{\circ} \mathrm{C}$ for $10 \mathrm{~min}, 40$ cycles of $95^{\circ} \mathrm{C}$ for $30 \mathrm{~s}, 63^{\circ} \mathrm{C}$ (mitochondria) or $58^{\circ} \mathrm{C}$ (SOX2) for $30 \mathrm{~s}$, and $72{ }^{\circ} \mathrm{C}$ for $90 \mathrm{~s}$. SYBR Green fluorescence was measured at the end of each extension step. Serial dilutions of a plasmid containing PCR products of the mitochondria and SOX2 genes were used as standards. Mitochondrial DNA copy number per gene was assessed as the expression ratio of mitochondria to SOX2, since SOX2 is a single-locus gene.

\section{ATP assay}

The ATP content of endometrial biopsies was assessed by measuring the luminescence generated in an ATP-dependent luciferin-luciferase bioluminescence assay (ATP Assay Kit. TOYO B-Net; Tokyo, Japan) according to the manufacturer's protocol. Samples were homogenized in buffer $(0.25 \mathrm{M}$ sucrose $10 \mathrm{mM}$ HEPES-NaOH, pH 7.4), and the luminescence of the supernatant was measured in duplicate using a LB96V Microplate Luminometer (Berthold Technologies; Wildberg, Germany). The ATP content per milligram of the sample was calculated based on the measured values.

\section{Quantification of SIRT1, PGC-1a, and BCL2L13 mRNA expression}

Total RNA was extracted from endometrial samples using the RNeasy Plus Universal Mini Kit (Qiagen) according to the manufacturer's instructions. Single-stranded cDNA was synthesized from $1 \mu \mathrm{g}$ of RNA using SuperScript II Reverse Transcriptase (Invitrogen) with an oligo-dT $12-18$ primer according to the manufacturer's instructions. qRT-PCR analysis was performed as described above. Primer sequences for SIRT1,PGC-1a, BCL2L13, and GAPDH are listed in Table S2. Thermocycling conditions were $95^{\circ} \mathrm{C}$ for 10 min, followed by 40 cycles of $95^{\circ} \mathrm{C}$ for $30 \mathrm{~s}, 60^{\circ} \mathrm{C}$ for $30 \mathrm{~s}$, and $72{ }^{\circ} \mathrm{C}$ for $90 \mathrm{~s}$. Serial dilutions of plasmids containing PCR products of each gene were used as standards. For each gene, its expression was normalized to GAPDH expression to adjust for any variation in the qRT-PCR.

\section{Microarray analysis of the bovine endometrium following parturition}

Endometrial tissues from ten female cows were collected twice from each individual at 42-86 (mean $60.1 \pm 4.0$ ) and $343-436$ (mean $388.9 \pm 8.2$ ) days after parturition. During each period, 7 days after 
estrus, endometrial tissue from the uterine horn ipsilateral to the corpus luteum (CL) was obtained using uterine biopsy forceps. RNA extraction and microarray analysis of samples with an RNA integrity number greater than 7.5 were performed as described above. Microarray data were deposited in the GEO database (accession number GSE107742).

\section{Experiment 2. Effect of resveratrol administration on the gene expression and pregnancy rate following embryo transfer in subfertile cows}

In subfertile cows, each uterine horn was injected with $25 \mathrm{~mL}$ of resveratrol $(100 \mu \mathrm{M}$; Sigma-Aldrich; St Louis, MO, USA) on day 1 ; endometrial tissue from the uterine horn, ipsilateral to the $\mathrm{CL}$, was biopsied on day 7. Additionally, to assess the effect of resveratrol on fertility, $25 \mathrm{~mL}$ of resveratrol $(100 \mu \mathrm{M})$ was injected into each uterine horn on day 1 , and embryo transfer to a recipient cow was performed on day 7 .

\section{Embryo transfer}

Embryos were collected nonsurgically from superovulating Japanese Black cattle on day 7 after standing estrus, as described previously ${ }^{56}$. Embryos classified as grade 1 or 2 blastocysts according to the IETS manual ${ }^{57}$ were cryopreserved using ethylene glycol as a cryoprotectant ${ }^{58}$. Day 7 embryos were thawed in a $25^{\circ} \mathrm{C}$ water bath, and each embryo was cultured in modified synthetic oviduct fluid supplemented with $1.5 \mathrm{mM}$ glucose and $5 \% \mathrm{FBS}$ at $38.5^{\circ} \mathrm{C}$ in $5 \% \mathrm{CO}_{2}, 5 \% \mathrm{O}_{2}$, and $90 \% \mathrm{~N}_{2}$ to confirm viability. After $6-12 \mathrm{~h}$ of culture, the quality of each viable embryo was reevaluated, and only grade 1 or 2 embryos were transferred to a uterine horn, ipsilateral to the $\mathrm{CL}$, of recipient cows. Pregnancy was confirmed by transrectal ultrasonography $30-80$ days after standing estrus.

\section{Statistical analysis}

Statistical analyses were conducted using JMP 9.0 software (SAS Institute Inc.; Cary, NC, USA). Statistically significant differences $(P<0.05)$ between fertile and subfertile cows in mitochondrial DNA copy number, ATP content of endometrial biopsies, and SIRT1, PGC-1a, and BCL2L13 mRNA expression were examined by Student's $t$ test. Plasma $\mathrm{P}_{4}$ concentrations in fertile and subfertile cows on days 0,5 , and 7 were analyzed for each day by Student's $t$ test. Statistically significant differences $(P<0.05)$ in the mRNA expression of SIRT1 and BCL2L13 between fertile, subfertile, and resveratrol-treated subfertile cows were determined by one-way ANOVA, followed by the Tukey HSD test.

\section{Data availability}

The datasets used in the current study are available from the corresponding author upon reasonable request.

\section{Declarations}

\section{Acknowledgements}


We thank Ms. H. Suzuki for providing technical assistance and the staff of the National Institute of Livestock and Grassland Science for their careful animal care. We thank Dr. Y. Nagamura and Ms. R. Motoyama (NIAS Microarray Open Lab) for their assistance with the microarray analysis. We thank Editage for their English language editing.

\section{Author Contributions}

S.M., H.I., and K.K. designed the research; S.M., S.N., S.M., M.S., N.T., T.S., and K.K. performed the research; S.M., S.N., S.M., and K.K. analyzed the data. S.M. and K.K. wrote the paper.

Competing interests: The authors declare no competing interests.

\section{References}

1 De Kruif, A. Factors influencing the fertility of a cattle population. J Reprod Ferti/54, 507-518 (1978).

2 Diskin, M. G. \& Sreenan, J. M. Fertilization and embryonic mortality rates in beef heifers after artificial insemination. J Reprod Ferti/59, 463-468 (1980).

3 Diskin, M. G. \& Morris, D. G. Embryonic and early foetal losses in cattle and other ruminants. Reprod Domest Anim43 Suppl 2, 260-267 (2008).

4 Sartori, R., Bastos, M. R. \& Wiltbank, M. C. Factors affecting fertilisation and early embryo quality in single- and superovulated dairy cattle. Reprod Fertil Dev22, 151-158, doi:10.1071/RD09221 (2010).

5 Dunne, L. D., Diskin, M. G. \& Sreenan, J. M. Embryo and foetal loss in beef heifers between day 14 of gestation and full term. Anim Reprod Sci58, 39-44 (2000).

6 Moraes, J. G. N. et al. Uterine influences on conceptus development in fertility-classified animals. Proc. Natl. Acad. Sci. U S A115, E1749-E1758, doi:10.1073/pnas.1721191115 (2018).

7 Ayalon, N. A review of embryonic mortality in cattle. J Reprod Fertil54, 483-493 (1978).

8 Matsuyama, S., Kojima, T., Kato, S. \& Kimura, K. Relationship between quantity of IFNT estimated by IFN-stimulated gene expression in peripheral blood mononuclear cells and bovine embryonic mortality after Al or ET. Reprod. Biol. Endocrinol. 10, 21, doi:10.1186/1477-7827-10-21 (2012).

9 Bazer, F. W. Uterine protein secretions: Relationship to development of the conceptus. J Anim Sci41, 1376-1382 (1975).

10 Roberts, R. M. \& Bazer, F. W. The functions of uterine secretions. J Reprod Ferti/82, 875-892 (1988).

11 Bazer, F. W., Wu, G., Johnson, G. A. \& Wang, X. Environmental factors affecting pregnancy: endocrine disrupters, nutrients and metabolic pathways. Mol Cell Endocrino/398, 53-68, doi:10.1016/j.mce.2014.09.007 (2014). 
12 Gray, C. A., Burghardt, R. C., Johnson, G. A., Bazer, F. W. \& Spencer, T. E. Evidence that absence of endometrial gland secretions in uterine gland knockout ewes compromises conceptus survival and elongation. Reproduction124, 289-300 (2002).

13 Gray, C. A. et al. Endometrial glands are required for preimplantation conceptus elongation and survival. Biol Reprod64, 1608-1613 (2001).

14 Ruiz-Gonzalez, I. et al. Exosomes, endogenous retroviruses and toll-like receptors: pregnancy recognition in ewes. Reproduction149, 281-291, doi:10.1530/REP-14-0538 (2015).

15 Spencer, T. E., Johnson, G. A., Bazer, F. W., Burghardt, R. C. \& Palmarini, M. Pregnancy recognition and conceptus implantation in domestic ruminants: roles of progesterone, interferons and endogenous retroviruses. Reprod Fertil Dev19, 65-78 (2007).

16 Katagiri, S. \& Takahashi, Y. Changes in EGF concentrations during estrous cycle in bovine endometrium and their alterations in repeat breeder cows. Theriogenology62, 103-112 (2004).

17 Forde, N. et al. Progesterone-regulated changes in endometrial gene expression contribute to advanced conceptus development in cattle. Biol Reprod81, 784-794 (2009).

18 Forde, N. et al. Changes in the endometrial transcriptome during the bovine estrous cycle: effect of low circulating progesterone and consequences for conceptus elongation. Biol Reprod84, 266-278 (2011).

19 Forde, N. et al. Effects of low progesterone on the endometrial transcriptome in cattle. Biol Reprod87, 124, doi:10.1095/biolreprod.112.103424 (2012).

20 Walker, C. G., Littlejohn, M. D., Mitchell, M. D., Roche, J. R. \& Meier, S. Endometrial gene expression during early pregnancy differs between fertile and subfertile dairy cow strains. Physiol Genomics44, 4758, doi:10.1152/physiolgenomics.00254.2010 (2012).

21 Salilew-Wondim, D. et al. Bovine pretransfer endometrium and embryo transcriptome fingerprints as predictors of pregnancy success after embryo transfer. Physiol Genomics42, 201-218, doi:10.1152/physiolgenomics.00047.2010 (2010).

22 Killeen, A. P. et al. Global gene expression in endometrium of high and low fertility heifers during the mid-luteal phase of the estrous cycle. BMC Genomics15, 234, doi:10.1186/1471-2164-15-234 (2014).

23 Hayashi, K. G. et al. Differential gene expression profiling of endometrium during the mid-luteal phase of the estrous cycle between a repeat breeder (RB) and non-RB cows. Reprod. Biol. Endocrinol. 15, 20, doi:10.1186/s12958-017-0237-6 (2017).

24 Geary, T. W. et al. Identification of Beef Heifers with Superior Uterine Capacity for Pregnancy. Biol Reprod95, 47, doi:10.1095/biolreprod.116.141390 (2016). 
25 Youle, R. J. \& van der Bliek, A. M. Mitochondrial fission, fusion, and stress. Science337, 1062-1065, doi:10.1126/science.1219855 (2012).

26 Weber, T. A. \& Reichert, A. S. Impaired quality control of mitochondria: aging from a new perspective. Exp Geronto/45, 503-511, doi:10.1016/j.exger.2010.03.018 (2010).

27 Puigserver, P. et al. A cold-inducible coactivator of nuclear receptors linked to adaptive thermogenesis. Cel/92, 829-839 (1998).

28 Nemoto, S., Fergusson, M. M. \& Finkel, T. SIRT1 functionally interacts with the metabolic regulator and transcriptional coactivator PGC-1\{alpha\}. J Biol Chem280, 16456-16460, doi:10.1074/jbc.M501485200 (2005).

29 Priault, M. et al. Impairing the bioenergetic status and the biogenesis of mitochondria triggers mitophagy in yeast. Cell Death Differ12, 1613-1621, doi:10.1038/sj.cdd.4401697 (2005).

30 Twig, G. et al. Fission and selective fusion govern mitochondrial segregation and elimination by autophagy. EMBO J27, 433-446, doi:10.1038/sj.emboj.7601963 (2008).

31 Zhang, Y. et al. The role of autophagy in mitochondria maintenance: characterization of mitochondrial functions in autophagy-deficient S. cerevisiae strains. Autophagy3, 337-346 (2007).

32 Mijaljica, D., Prescott, M. \& Devenish, R. J. Different fates of mitochondria: alternative ways for degradation? Autophagy3, 4-9 (2007).

33 Narendra, D., Tanaka, A., Suen, D. F. \& Youle, R. J. Parkin is recruited selectively to impaired mitochondria and promotes their autophagy. J Cell Bio/183, 795-803, doi:10.1083/jcb.200809125 (2008).

34 Nan, J. et al. Molecular regulation of mitochondrial dynamics in cardiac disease. Biochim Biophys Acta1864, 1260-1273, doi:10.1016/j.bbamcr.2017.03.006 (2017).

35 Chang, J. Y., Yi, H. S., Kim, H. W. \& Shong, M. Dysregulation of mitophagy in carcinogenesis and tumor progression. Biochim Biophys Acta1858, 633-640, doi:10.1016/j.bbabio.2016.12.008 (2017).

$36 \mathrm{Kim}$, I., Rodriguez-Enriquez, S. \& Lemasters, J. J. Selective degradation of mitochondria by mitophagy. Arch Biochem Biophys462, 245-253, doi:10.1016/j.abb.2007.03.034 (2007).

37 Kanki, T., Wang, K., Cao, Y., Baba, M. \& Klionsky, D. J. Atg32 is a mitochondrial protein that confers selectivity during mitophagy. Dev Cel/17, 98-109, doi:10.1016/j.devcel.2009.06.014 (2009).

38 Okamoto, K., Kondo-Okamoto, N. \& Ohsumi, Y. Mitochondria-anchored receptor Atg32 mediates degradation of mitochondria via selective autophagy. Dev Cel/17, 87-97, doi:10.1016/j.devcel.2009.06.013 (2009). 
39 Murakawa, T. et al. Bcl-2-like protein 13 is a mammalian Atg32 homologue that mediates mitophagy and mitochondrial fragmentation. Nat Commun6, 7527, doi:10.1038/ncomms8527 (2015).

$40 \mathrm{Hara}, \mathrm{H}$. et al. Mitochondrial fragmentation in cigarette smoke-induced bronchial epithelial cell senescence. Am J Physiol Lung Cell Mol Physio/305, L737-746, doi:10.1152/ajplung.00146.2013 (2013).

41 Correia-Melo, C. et al. Mitochondria are required for pro-ageing features of the senescent phenotype. EMBO J35, 724-742, doi:10.15252/embj.201592862 (2016).

42 Korolchuk, V. I., Miwa, S., Carroll, B. \& von Zglinicki, T. Mitochondria in Cell Senescence: Is Mitophagy the Weakest Link? EBioMedicine21, 7-13, doi:10.1016/j.ebiom.2017.03.020 (2017).

43 Dalle Pezze, P. et al. Dynamic modelling of pathways to cellular senescence reveals strategies for targeted interventions. PLoS Comput Bio/10, e1003728, doi:10.1371/journal.pcbi.1003728 (2014).

44 Garcia-Prat, L. et al. Autophagy maintains stemness by preventing senescence. Nature529, 37-42, doi:10.1038/nature16187 (2016).

45 Tanaka, H. et al. The SETD8/PR-Set7 Methyltransferase Functions as a Barrier to Prevent SenescenceAssociated Metabolic Remodeling. Cell Rep18, 2148-2161, doi:10.1016/j.celrep.2017.02.021 (2017).

46 Nishimura, K. et al. Perturbation of ribosome biogenesis drives cells into senescence through 5S RNPmediated p53 activation. Cell Rep10, 1310-1323, doi:10.1016/j.celrep.2015.01.055 (2015).

47 Kaplon, J. et al. A key role for mitochondrial gatekeeper pyruvate dehydrogenase in oncogene-induced senescence. Nature498, 109-112, doi:10.1038/nature12154 (2013).

48 Hutter, E. et al. Senescence-associated changes in respiration and oxidative phosphorylation in primary human fibroblasts. Biochem J380, 919-928, doi:10.1042/bj20040095 (2004).

49 Quijano, C. et al. Oncogene-induced senescence results in marked metabolic and bioenergetic alterations. Cell Cycle11, 1383-1392, doi:10.4161/cc.19800 (2012).

50 Takebayashi, S. et al. Retinoblastoma protein promotes oxidative phosphorylation through upregulation of glycolytic genes in oncogene-induced senescent cells. Aging Cel/14, 689-697, doi:10.1111/acel.12351 (2015).

51 Dorr, J. R. et al. Synthetic lethal metabolic targeting of cellular senescence in cancer therapy. Nature501, 421-425, doi:10.1038/nature12437 (2013).

52 Baur, J. A. \& Sinclair, D. A. Therapeutic potential of resveratrol: The in vivo evidence. Nat Rev Drug Discov5, 493-506, doi:10.1038/nrd2060 (2006).

$53 \mathrm{Wu}, \mathrm{Y}$. et al. Resveratrol-activated AMPK/SIRT1/autophagy in cellular models of Parkinson's disease. Neurosignals19, 163-174, doi:10.1159/000328516 (2011). 
54 Ishiguro, S. et al. Low Temperature-Responsive Changes in the Anther Transcriptome's Repeat Sequences Are Indicative of Stress Sensitivity and Pollen Sterility in Rice Strains. Plant Physiology164, 671-682 (2014).

55 Tatebayashi, R. et al. Gene-expression profile and postpartum transition of bovine endometrial side population cells. Biol Reprod, doi:10.1093/biolre/ioab (2021).

56 Kimura, K. et al. Successful superovulation of cattle by a single administration of FSH in aluminum hydroxide gel. Theriogenology68, 633-639 (2007).

57 Stringfellow, D. A. \& Seidel, S. M. Manual of the International Embryo Transfer Society: a procedural guide and general information for the use of embryo transfer technology emphasizing sanitary procedures. International Embryo Transfer Society3rd ed., Savoy, IL (1998).

58 Dochi, O. et al. Direct transfer of bovine embryos frozen-thawed in the presence of propylene glycol or ethylene glycol under on-farm conditions in an integrated embryo transfer program. Theriogenology49, 1051-1058 (1998).

\section{Tables}

Due to technical limitations, table 1-3 is only available as a download in the Supplemental Files section.

\section{Figures}


(a)

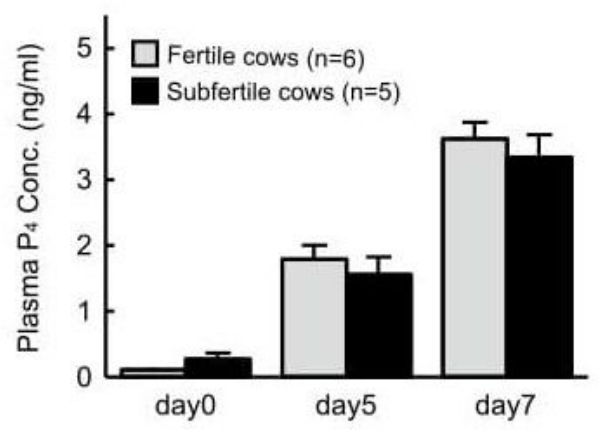

(b)
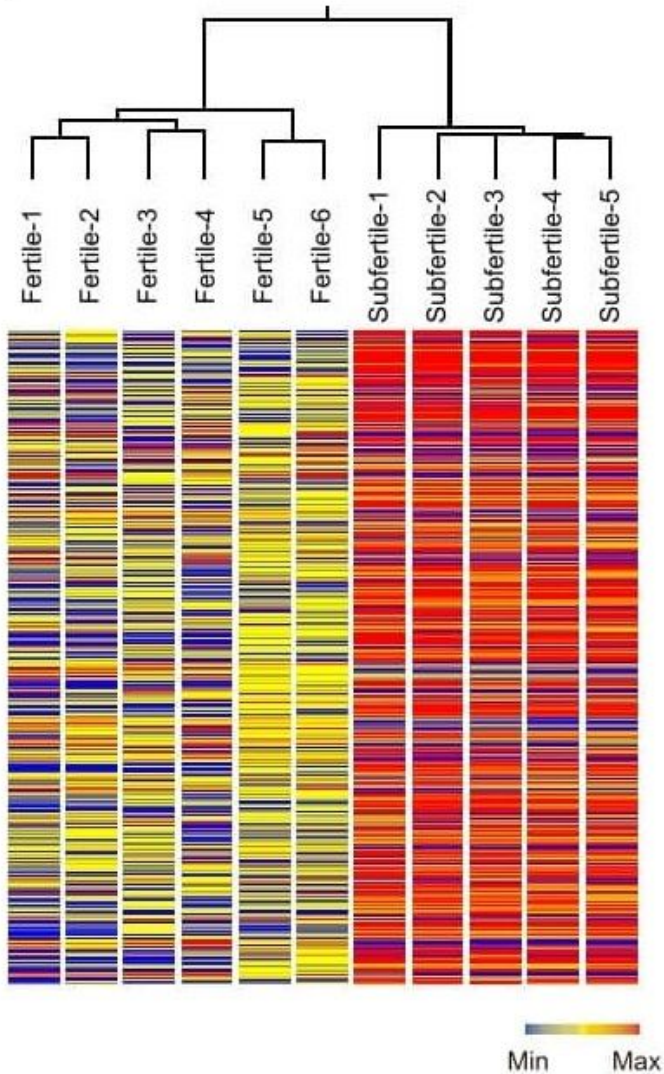

(c)

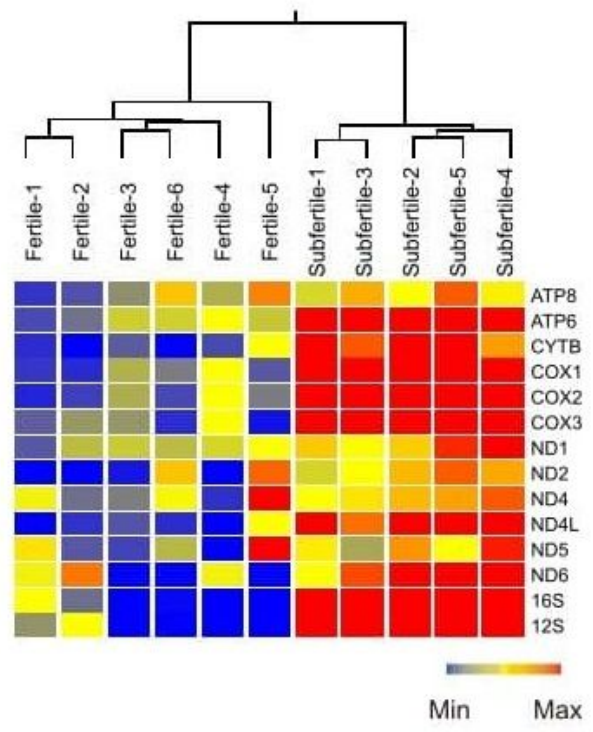

(d)

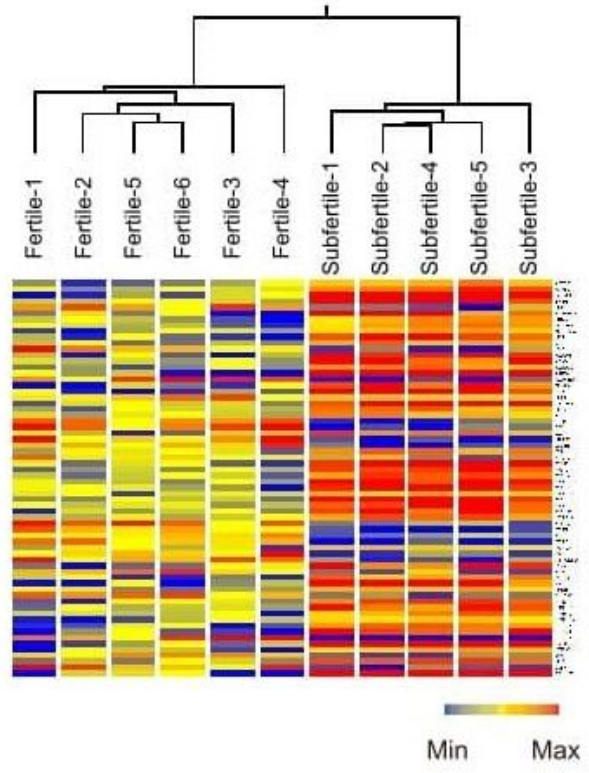

Figure 1

Plasma P4 concentrations and global gene expression profiles in the endometrium of fertile and subfertile cows. (a) Plasma P4 concentrations on days 0,5 , and 7 in fertile and subfertile cows. (b) Heat map of all genes in fertile and subfertile cows. Hierarchical clustering of microarray data classified cows into two major clusters. (c) Heat maps of genes encoded by mitochondrial DNA and (d) nuclear genes encoding mitochondrial proteins in fertile and subfertile cows. 
(a)

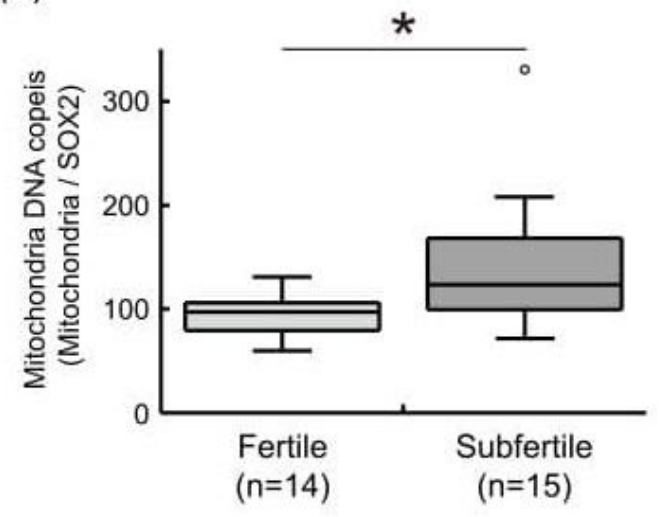

(b)

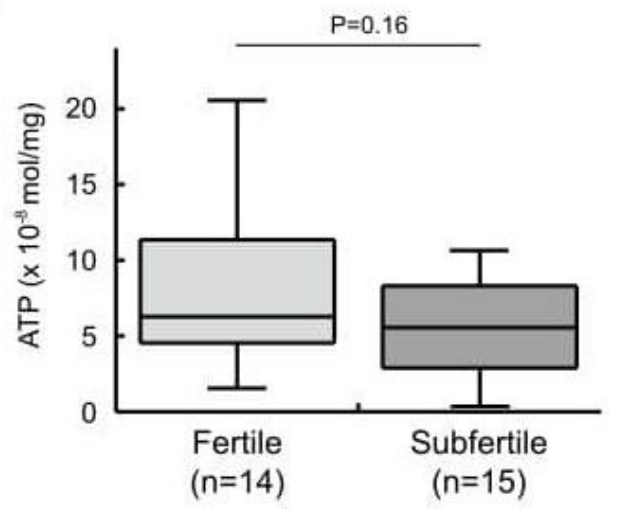

\section{Figure 2}

Mitochondrial DNA copy number and ATP content in the endometrium of fertile and subfertile cows. (a) Comparison of mitochondrial DNA copy number determined by qPCR analysis and (b) ATP content per milligram of biopsied endometrium from fertile cows and subfertile cows. Values represent means \pm SEM. Data were analyzed using Student's t-test. *Significant difference compared to fertile cows $(\mathrm{P}<$ 0.05).

(a)

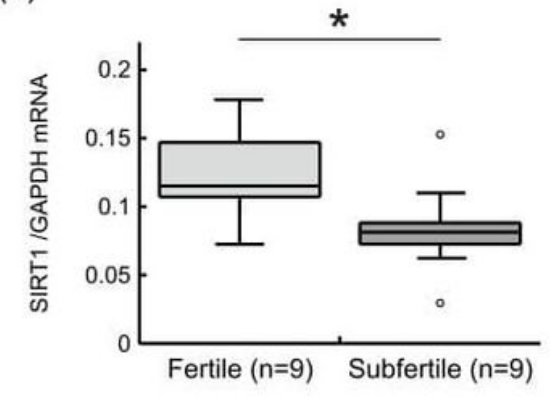

(b)

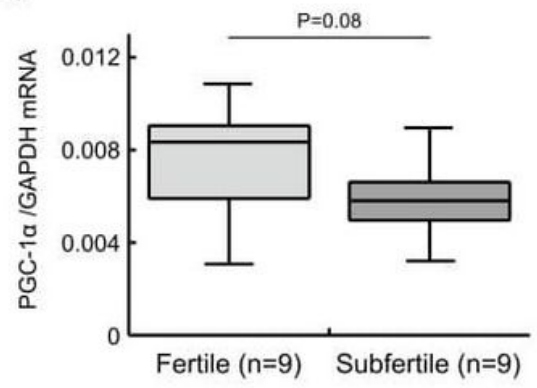

(c)

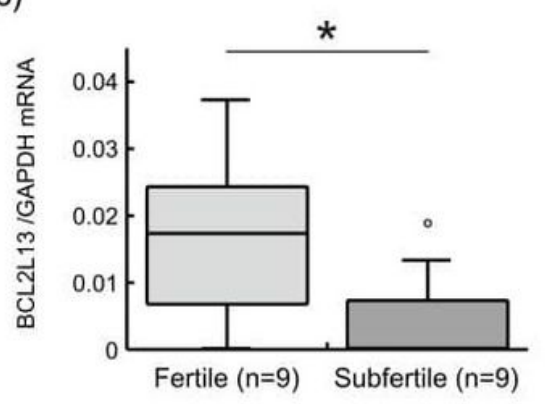

Figure 3 
SIRT1, PGC-1a, and BCL2L13 mRNA expressions in the endometrium of fertile and subfertile cows. (a) Comparison of relative mRNA expression of SIRT1, (b) PGC-1a, and (c) BCL2L13 determined by qRT-PCR analysis of endometrial biopsies from fertile and subfertile cows. Values are means \pm SEM. Data were analyzed using Student's t-test. *Significant difference compared to fertile cows $(P<0.05)$.

(a)

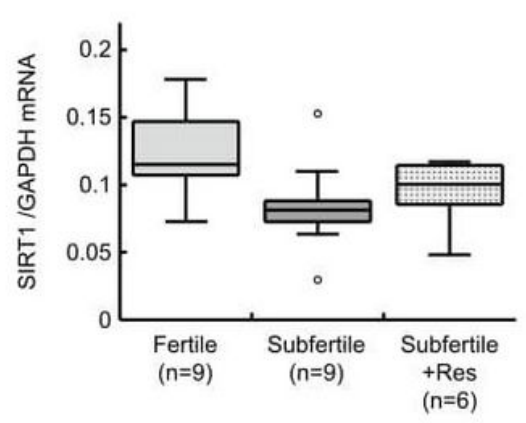

(b)

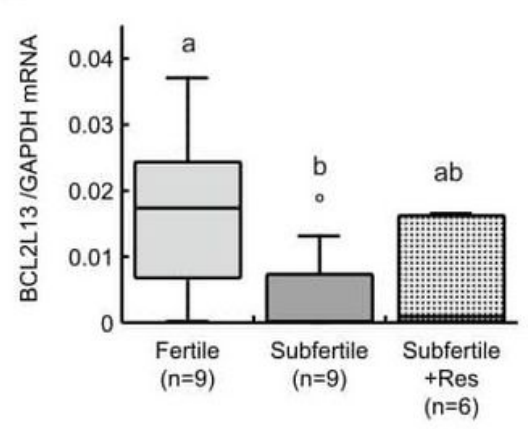

(c)

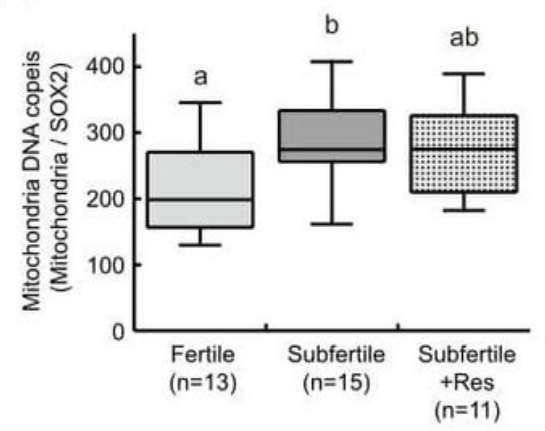

\section{Figure 4}

SIRT1 and BCL2L13 mRNA expressions and mitochondrial DNA copy number in the endometrium of fertile cows, subfertile cows and subfertile cows with resveratrol. (a) Comparison of relative mRNA expression of SIRT1 and (b) BCL2L13 determined by qRT-PCR analysis and (c) mitochondrial DNA copy number by qPCR analysis of endometrial biopsies from fertile cows, subfertile cows, and subfertile cows administered resveratrol $(100 \mu \mathrm{M}$, Subfertile+Res). Values are means \pm SEM. Values with different letters indicate significant differences ( $P<0.05$, one-way ANOVA followed by Tukey's post-hoc tests).

\section{Supplementary Files}

This is a list of supplementary files associated with this preprint. Click to download.

- SupplementaryInformationS.MatsuyamaNSR210210.pdf

- Table1S.MatsuyamaNSR210120.xIsx

- Table2S.MatsuyamaNSR210120.xlsx

- Table3S.MatsuyamaNSR210120.xlsx 